\title{
Type III secretion system confers enhanced virulence in clinical non-O1/non- O139 Vibrio cholerae
}

Samia Zeb" ${ }^{1, \#}$, Muhammad Ali Shah ${ }^{1, \#, ~ M u h a m m a d ~ Y a s i r ~}{ }^{1}$, Hassaan Mehboob Awan ${ }^{1}$, Peerada Prommeenate $^{2}$, Amornpan Klanchui ${ }^{2}$, Brendan W. Wren ${ }^{3}$, Nicholas Thomson ${ }^{3,4}$, Habib Bokhari ${ }^{1, *}, \#$

${ }^{1}$ Department of Biosciences, COMSATS University Islamabad (CUI), Islamabad, Pakistan.

${ }^{2}$ Biochemical Engineering and Pilot Plant Research and Development (BEC) Unit, National Center for Genetic Engineering and Biotechnology, National Science and Technology Development Agency, King Mongkut's University of Technology Thonburi, Bangkok 10150, Thailand

${ }^{3}$ Department of Pathogen Molecular Biology, London School of Hygiene \& Tropical Medicine, UK

${ }^{4}$ Wellcome Trust Sanger Institute, Cambridge

*Corresponding Author: habib@comsats.edu.pk

\# These authors contributed equally to this work.

\section{Abstract}

Vibrio cholerae $\mathrm{O} 1$ infections mainly are responsible for significant mortality and morbidity amongst children, however, non-O1/non-O139 V. cholerae have also been reported to cause mild to severe infections because of their virulence potential. The pathogenic mechanisms of non-O1, non-O139 isolates are not as clearly understood as for that of $\mathrm{O} 1$ and $\mathrm{O} 139$ isolates. Type three secretion system (TTSS) is also considered one of the important virulent factors and during the current study, we investigated the role of TTSS in association with non-O1/non-O139 clinical isolates. We report that the presence of TTSS in non-O1/non-O139 V. cholerae clinical isolate (D13) from a child confers more virulence compared to the one lacking it (D15) in another clinical case during the small cholera epidemic. Moreover, the antibiotic susceptibility profiles of D13 and D15 indicate that they are multiple drug resistance (MDR) isolates. The sequence analysis for TTSS cluster was carried out for D13 and compared with the TTSS positive 
reference Vibrio parahaemolyticus RIMD2210633 and V. cholerae AM19226 non-O1/nonO139. Furthermore, the pathogenic potential of D13 \& D15 was also explored in simple and economical invertebrate host model, Galleria mellonella and the results revealed that TTSS $^{+v e}$ isolate (D13) was more virulent compared to $\mathrm{TTSS}^{-\mathrm{ve}}$ isolate (D15). We suggest that this distinct genetic difference, seen in natural variants D13 and D15, is also reflected by the clinical picture of the former in contributing towards the severity of disease symptoms and this finding was further validated by assessing virulence potential of both isolates using inexpensive $G$. mellonella infection model.

\section{Introduction}

Vibrio cholerae, a gram-negative bacterium, belonging to the r-subdivision of the family proteobacteriaceae causes acute watery diarrheal disease known as cholera [1]. Cholera has claimed millions of lives in the past, but today cholera is still endemic in Africa and South Asia where it has been reported for at least 1,000 years [2]. Cholera is mostly a disease of the tropics, it is often associated with low quality sanitation management where the people had limited access to microbiologically safe drinking water. Natural calamities such as floods, earthquakes, and political disturbances resulting in wars, also increase the likelihood of a cholera epidemic. $V$. cholerae is a highly heterogenous species, based on somatic $\mathrm{O}$ antigen $>200$ serogroups exists, the majority of which are non-pathogenic and only O1 and O139 out of 200 serogroups are known to cause widespread and repeated epidemics. The $\mathrm{O} 1$ and $\mathrm{O} 139$ can be further subdivided into two biotypes, El Tor and classical [3-4].

Seven cholera pandemics have been recorded since 1817 . It is widely accepted that the first six epidemics are caused by biotype: classical, while the current seventh pandemic was due to E1 Tor. Interestingly, recent studies indicate that non-O1/non-O139 V. cholerae are 
increasingly recognized as a cause of sporadic cases of gastroenteritis usually resulting in severe bloody diarrhea and are also associated with extra-intestinal infections including bacteremia, skin and wound infections in immuno-compressed individuals [5-6].

V. cholerae O1, O139, and non-O1/non-O139 serogroups differ in their clinical outcome because of the number of virulence factors ranging from array of toxins, colonization factors, antibiotic resistance, resistance to disinfectant provided by capsular polysaccharides, as well as unique surface antigens (O139 lipopolysaccharide and O antigen capsule) [7-10].

In different parts of Africa, USA, Asia, and Europe cholera outbreaks were recorded and their studies [11-12] revealed that $V$. cholerae non-O1/non-O139 strains are the causative agent of these outbreaks. Some of these isolates although non-pandemic serogroups possess known genes/systems associated with virulence including the cholera toxin phage (CTX), genomic islands VSP-1 and 2, and VPI-1 and 2. However, even without typically associated virulence factors with O1 and O139 serogroup isolates such as cholera toxin (CT), carried by CTX and TCP genes required for human intestinal colonization, additional virulence factors comprising heat-stable enterotoxin (ST), haemolysin (HlyA), mannose-sensitive hemagglutinin (MSHA) pilus, repeats in toxin (RTX), and the type three secretion system (TTSS) enhance virulence of non-agglutinating vibrios (NAGs) i.e. non-O1/non-O139 isolates [13-17].

Previous studies revealed that the non-O1/non-O139 V. cholerae strain AM-19226 successfully colonized the infant mouse intestine as well as induced a cholera-like disease condition in adult rabbits [17-18]. The genome analysis of AM-19226 further revealed that it lacked $\mathrm{CT}$ and TCP suggesting that perhaps other virulence factors including type III secretion system (TTSS) may have compensated for the lack of CT and TCP in infant mouse and rabbit models [17]. TTSS has been reported from a wide spectrum of gram-negative bacteria including 
non-O1/non-O139 $V$. cholerae isolates of both clinical and environmental origins [19-20, 2324]. They facilitate direct translocation of TTSS secreted effectors proteins through a syringelike a needle into the host cytosol [19-20]. The translocated effector proteins are known to interfere cellular processes including depolymerization of cytoskeleton components [21] and more specifically the products of factors such as $o p \mathrm{~F}$, vop E, stimulates actin nucleation and promotes actin depolymerization respectively [22].

The genome analysis of non-O1/non-O139 TTSS shows strong homology to $V$. parahaemolyticus TTSS gene clusters [17] and have been proposed to play a role in virulence and environmental adaptation of these isolates [17]. However, the significance of TTSS particularly in association with human non-O1/non-O139 V. cholerae and other less characterized effectors in the pathogenesis of the diarrheal disease is not well understood.

We identified two non-O1/non-O139 V. cholerae isolates from children under 10 years of age showing cholera-like symptoms and, investigated their susceptibility profiles as well the virulence potential of the isolates with a focus on TTSS. This is the first report where TTSS in association with non-O1/non-O139 V. cholerae clinical cases was investigated from Pakistan and we believe a greater understanding of the differences between pandemic and non-pandemic serogroups will have implications for our better monitoring and surveillance of $V$. cholerae in the future.

\section{Material and methods}

\subsection{Ethical statement}

This study was first approved by an ethical board of the Department of Biosciences, CUI, Islamabad. 


\subsection{Sample collection and identification:}

Suspected cholera stool samples were collected in Cary-Blair transport medium within 24 hours of the patient being admitted and transported to Microbiology \& Public Health Laboratory at Department of Biosciences, COMSATS University - Islamabad. For enrichment of samples, $1 \mathrm{x}$ alkaline peptone water was used after $6-8 \mathrm{hrs}$ incubation at $37^{\circ} \mathrm{C}$. After enrichment, the inoculum was taken and smeared on solid thiosulfate citrate bile salts (TCBS) sucrose medium and then it was placed at $37^{\circ} \mathrm{C}$ for $16-20 \mathrm{hrs}$ and yellowish-green colonies, characteristic of Vibrio species, were selected and biochemical and molecular tests were performed as described previously [21-22]. Isolates were preserved in $\mathrm{BHI}$ broth at $-8^{\circ} \mathrm{C}$ containing $20 \%$ glycerol.

\subsection{Serotyping determination}

Slide agglutination test was carried out to confirm if the isolates belonged to O1 and O139 serogroups or non-O1/non-O139. Monovalent Inaba and Ogawa antisera and Polyvalent Inaba and Ogawa antisera (Denka Seiken, Japan) were used to determining serogroup O1. The serogroup specific encoding regions were amplified by multiplex PCR [25].

\subsection{Antimicrobial susceptibility test}

According to CLSI guideline (CLSI, 2009) [26], antimicrobial susceptibility to different antibiotics was checked by Kirby Bauer disc diffusion tests on Muller-Hinton Agar (Oxoid). The antibiotics used were: trimethoprim $(25 \mu \mathrm{g})$, tetracycline $(30 \mu \mathrm{g})$, ampicillin $(10 \mu \mathrm{g})$, sulfamethoxazole/trimethoprim $(23.71 / 1.25 \mu \mathrm{g})$, erythromycin $(15 \mu \mathrm{g})$, streptomycin $(10 \mu \mathrm{g})$, ofloxacin $(5 \mu \mathrm{g})$, nalidixic acid $(30 \mu \mathrm{g})$, ceftazidime $(30 \mu \mathrm{g})$, ciprofloxacin $(5 \mu \mathrm{g})$, cefotaxime $(30 \mu \mathrm{g})$, and chloramphenicol $(30 \mu \mathrm{g})$. As a susceptible control, Escherichia coli ATCC 25922 were used. 


\subsection{DNA extraction and genomic characterization of D13 and D15}

Overnight culture of $V$. cholerae D13 (isolate from 9 year old child) and D15 (isolate from 10 year old child) isolates in nutrient broth was used to extract DNA by ArchiPure DNA extraction Kit (Prime 5, UK). PCR was performed to screen for $r t x A$ and $o m p W$ [27, 28]. Further, screening through PCR using various gene specific primers was carried out for detecting virulence factors i.e. $t c p A, \operatorname{ctx} A$, TTSS (vcs $C, v s p D, v c s V)$ (Table 1).

\subsection{Genetic characterization of antibiotic resistance}

The isolated strains were analyzed by PCR to detect conjugative and integrative element SXT [29], integrons [30], and genetic basis for resistance to sulfamethoxoles [31-32], fluoroquinolones [33-34], streptomycin [35-36], chloramphenicol [36] and trimethoprim.

Table 1: Primers used in the study

\begin{tabular}{|c|c|c|c|c|}
\hline Gene & Primer sequence (5' to 3') & $\begin{array}{l}\text { Annealing } \\
\text { Temp. }\left({ }^{\circ} \mathrm{C}\right)\end{array}$ & $\begin{array}{l}\text { Product } \\
\text { (bps.) }\end{array}$ & References \\
\hline$r t x A$ & $\begin{array}{l}\text { F: AGCAAGAGCATTGTTGTTCCTACC } \\
\text { R: ACTTCCCTGTACCGCACTTAGAC }\end{array}$ & 61 & 120 & {$[27]$} \\
\hline ompW & $\begin{array}{l}\text { F: CACCAAGAAGGTGACTTTATTGTG } \\
\text { R: GAACTTATAACCACCCGCG }\end{array}$ & 61 & 588 & {$[28]$} \\
\hline $\operatorname{ctx} A$ & $\begin{array}{l}\text { F: CTCAGACGGGATTTGTTAGGCACG } \\
\text { R: TCTATCTCTGTAGCCCCTATTACG }\end{array}$ & 60 & 301 & {$[37]$} \\
\hline O1 rfb & $\begin{array}{l}\text { F: GTTTCACTGAACAGATGGG } \\
\text { R: GGTCATCTGTAAGTACAAC }\end{array}$ & 55 & 192 & {$[25]$} \\
\hline O139 rfb & $\begin{array}{l}\text { F: AGCCTCTTTATTACGGGTGG } \\
\text { R: GTCAAACCCGATCGTAAAGG }\end{array}$ & 55 & 449 & {$[25]$} \\
\hline $\operatorname{tcp} A^{C l}$ & $\begin{array}{l}\text { F: CACGATAAGAAAACCGGTCAAGAG } \\
\text { R: ACCAAATGCAACGCCGAATGGAGC }\end{array}$ & 60 & 472 & {$[37]$} \\
\hline$t c p A^{E T}$ & $\begin{array}{l}\text { F: GAAGAAGTTTGTAAAAGAAGAACAC } \\
\text { R: GAAAGGACCTTCTTTCACGTTG }\end{array}$ & 60 & 672 & {$[37]$} \\
\hline$v c s \mathrm{C} 2$ & $\begin{array}{l}\text { F:CGTCGACGTTACCGATGCTATGGGT } \\
\text { R:GCTCTAGAAGTCGGTTGTTTCGGTAA }\end{array}$ & 60 & 535 & {$[42]$} \\
\hline$v c p \mathrm{D}$ & $\begin{array}{l}\text { F:CGTCGACAGTTGAGCCAATTCCATT } \\
\text { R:AGACGACCAAACGAGATAATG }\end{array}$ & 55 & 422 & {$[17]$} \\
\hline$v c s \mathrm{~V} 2$ & $\begin{array}{l}\text { F:ATGCAGATCTTTTGGCTCACTTGATGG } \\
\text { R:ATGCGTCGACGCCACATCATTGCTTGC }\end{array}$ & 60 & 535 & {$[17]$} \\
\hline
\end{tabular}




\subsection{Genome sequencing of D13 and D15}

D13 and D15 isolates were sequenced by Illumina HiSeq platform. Initially by creating multiplex sequencing libraries were created each with 250 bps capacity. To get paired end reads of 72 bases of 96 libraries, libraries were loaded on the sequencing platform cell. Unique index tagging was used to labeled each library. For both samples 200X- average coverage was achieved and using a de novo genome assembly program Velvet version 0.7 [44], the 72 bps paired end reads were assembled. For best k-mer size and at least $20 \times k-m e r$ coverage, parameters were set to appropriate values. By using SMALT software, genome of $V$. cholerae O1 El Tor strain N16961 is mapped to the 72-base paired end read data of both isolates and whole genome alignment of both isolates was obtained. The data was used to predict and compare TTSS cluster with reference sequences (RIMD2210633; AM19226).

\subsection{Type three secretion system (TTSS) prediction and comparison}

The TTSS gene cluster in $V$. cholerae D13 and D15 genome sequences were predicted using BLAST [45]. $V$. cholerae AM19226 was taken as the reference sequence against which $V$. cholerae D13 and D15 genomes were queried. BLAST comparisons among the multiple genomic regions of interest were further visualized using Easyfig [46].

\subsection{Genome Comparison of D13 \& D15}

To do the annotation of both D13 and D15, the genome sequences of both strains had been submitted through the SEED-RAST server. 


\subsection{Galleria mellonella infection model for clinical non-O1/non-O139 V. Cholera}

To investigate the virulence potential of TTSS positive and negative clinical non-O1/nonO139 V. cholerae isolates, infection assays were performed by using an invertebrate model system, Galleria mellonella as described previously [47, 48]. Briefly, $10 \mu \mathrm{l}$ taken from overnight $(\mathrm{O} / \mathrm{N})$ culture, grown at $30^{\circ} \mathrm{C}$ carrying $10^{4} \mathrm{CFU}$ to $10^{8} \mathrm{CFU}$ were injected into first right proleg of G. melonella larvae. 10 larvae were injected in each case. $70 \%$ (vol $/ \mathrm{vol})$ ethanol was used for surface disinfection, and experimental models were scored at 24 and 48 hours for phenotypes such as survival, movement and change in color. In parallel, PBS diluent was injected to control larvae and the experiment considered successful when no control larvae died. Larvae which show no movement/response when touched were considered dead. Data from at least three replicates was analyzed and for each strain, 50\% lethal doses were calculated.

\section{Results}

\subsection{Clinical manifestations of TTSS ${ }^{+}$and TTSS non-O1/non-O139 isolates}

This study is based on two child patients presenting cholera-like illness. On the basis of standard biochemical tests and PCR, stool sample of both patients were considered positive for presence of $V$. cholerae [28, 49]. Briefly, cholera isolate-D13 was isolated from a 9 year old male patient admitted to pediatric ward of civil hospital, Dera Ismail Khan (Khyber Pukhtoon Khwa province) with symptoms of watery stool accompanied with fever $\left(102^{\circ} \mathrm{F}\right)$, concomitant with episodes of vomiting, severe dehydration and frequent passage of watery stools (IsolateD13). The second isolate (Isolate-D15) was taken from a 10 year old male patient with profuse watery stool and other gastroenteritis/diarrhea like symptoms(Isolate-D15). 
Serogroup determination by using slide agglutination test revealed that $V$. cholerae isolates (D13 and D15) belonged to non-O1/non-O139 serogroups as they lack O1rfb/O139rfb or

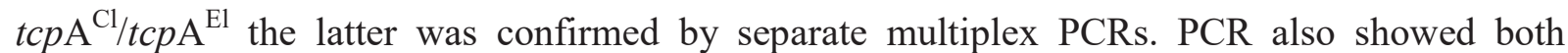
isolates lacked the cholera toxin and the cholera prophage encompassing genes $\operatorname{ctx} A$, zot, ace and RS1. These isolates also lacked tcpA, an important virulence factor located on vibrio pathogenicity island -1. However, using TTSS gene -specific primers (Table 1), D13 was found positive for TTSS (Figure 1.)



Figure 1: Detection of TTSS in D-13.

\subsection{Genotypic and Phenotypic characterisation of antimicrobial resistance in D13 and D15}

D13 and D15 were multiple drug resistant as they exhibited resistance phenotypes for streptomycin, co-trimoxazole, nalidixic acid and ciprofloxacin on Mueller-Hinton (MH) agar. However, resistance to ceftazidime and ofloxacin varied as D13 was resistant to ceftazidime whereas D15 to ofloxacin. Response to tetracycline, erythromycin and ceftaxime were intermediate or sensitive in both isolates. 
Integrons of all three classes were absent in D13 and D15 as consensus sequences of their integrases were not amplified by PCR. Integrative and conjugative element, SXT was not found in these isolates. $\operatorname{str} A$ and $\operatorname{str} B$ (encoding streptomycin resistance) were present as the PCR revealed amplification of 284 and 248 bps fragments respectively whereas in case of sulfamethoxazole resistance encoding genes, sul2 was present and sull and sul3 were absent. Furthermore, qnrA, qnrB and qnrS (fluoroquinoles resistance) were not present in D13 and D15. Among, other antibiotic resistance encoding genes, florR (chloramphenicol resistance) was only present in D15. (Table 2)

Table 2: Antibiotic resistance characterization

\begin{tabular}{|c|c|c|}
\hline $\begin{array}{l}\text { Non-O1/non- } \\
\text { O139 } V \text {. } \\
\text { cholera }\end{array}$ & $\begin{array}{l}\text { Antimicrobial resistance } \\
\text { encoding genes present }\end{array}$ & $\begin{array}{l}\text { Antimicrobial resistance encoding elements not } \\
\text { detected }\end{array}$ \\
\hline D13 & sul2, strA, strB & $\begin{array}{l}\text { SXT, dfrA1, dfrA18, tetA, , qnrA, qnrB, qnrS, sul1, sul3, } \\
\text { intI, int2, int } 3 \text {, florR }\end{array}$ \\
\hline D15 & sul2, str $A$, str $B$, florR & $\begin{array}{l}\text { SXT, dfrA } 1, \text { dfra18, tetA, qnrA, qnrB, qnrS, sull, sul3, } \\
\text { intI, int } 2, \text { int } 3 .\end{array}$ \\
\hline
\end{tabular}

\subsection{TTSS comparative sequence analysis}

The conserved TTSS gene cluster in the assembled completed genomes of $V$. cholerae D13 and D15 was identified based on similarity searches through BLAST. The alignment results confirmed the existence of TTSS cassette in D13 isolate while it was not found in the genome D15 isolate. Comparative analysis results of the conserved TTSS genes cluster of $V$. parahaemolyticus RIMD2210633, V. cholerae AM19226, and V. cholerae D13 were visualized and shown in Figure 2. The result from TTSS gene cluster genome alignment has also shown the difference in gene organization of conserved TTSS gene cluster in all these strains. 


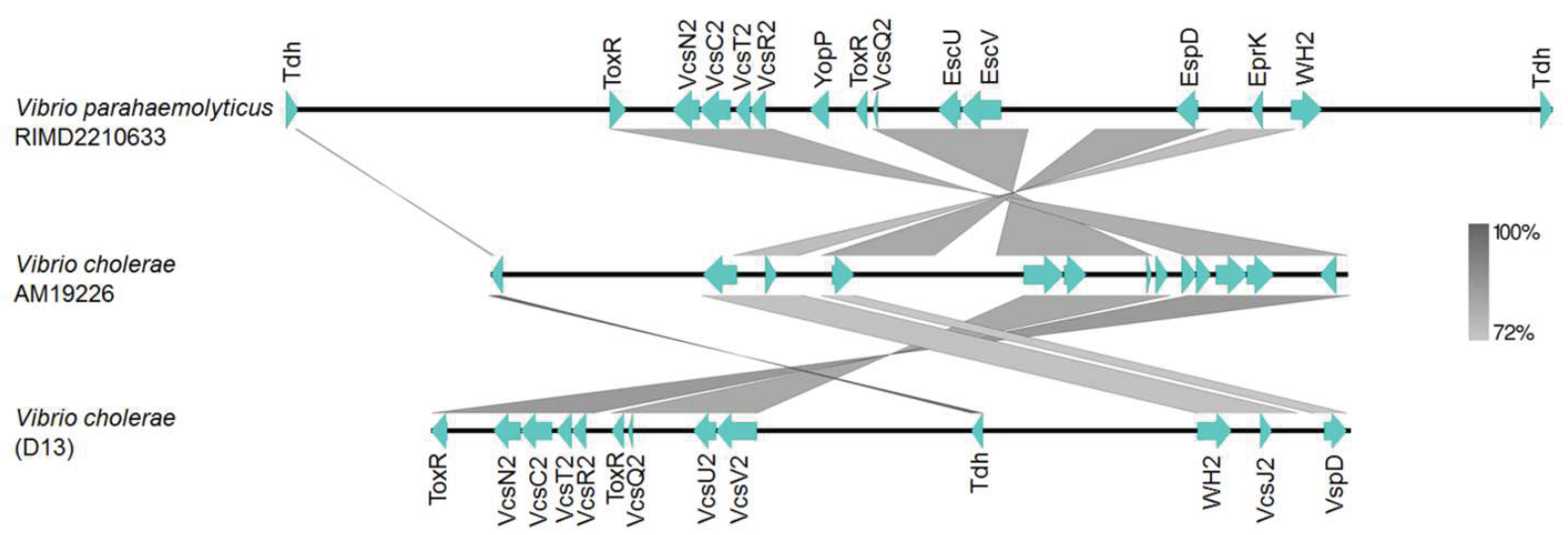

Figure 2. Comparison of TTSSs gene cluster of genomes of $V$. parahaemolyticus RIMD2210633, V. cholerae AM19226, and $V$. cholerae D13. Arrows represent TTSS CDSs which comes through BLAST hits and annotation. Vertical lines between sequences show regions having shared homology .

\subsection{Genome Comparison of D13 \& D15}

In general, both genomes (D13 and D15) showed the similar number of genes that have been annotated. The genome statistic also showed the similar characters. The results of analysis are shown as in Appendix A (Figures A1 \& A2).

\subsection{Mortality in G. mellonella infected with V. cholerae TTSS $^{+v e}$ isolate and TTSS ${ }^{-v e}$ isolate}

G. mellonella is injected with a range of $10^{4}$ to $10^{8} \mathrm{CFU}$ of bacterial cells (D-15 and D13) and number of surviving insects were recorded after $24 \mathrm{hrs}$ (A) and $48 \mathrm{hrs}$ (B), and are shown in tabular form under the graph. The numbers (CFUs) shown in these results are the mean values of three independent experiments.

Keeping in consideration the different clinical manifestations shown by TTSS $^{+\mathrm{ve}}$ (D13) and $\mathrm{TTSS}^{-\mathrm{ve}}$ (D15) isolates, we investigated their pathogenicity in G. mellonella larvae. In the first right proleg of larvae, $10^{4}$ to $10^{8} \mathrm{CFUs}$ of bacterial cells (D15, D13) were injected, and their survival was monitored for 24 hours and 48 hours. The virulence potential of D13 and D15 was 
compared with PBS injected control group. No mortality of G. mellonella larvae was observed in control group. Death of at least $90 \%$ larvae was observed due to presence of TTSS $^{+\mathrm{ve}}$ strain.

On the other hand, larvae infected with TTSS $^{-v e}$ strain showed $60 \%$ deaths at $10^{4}$ CFUs, 24 hours post injection (Figure 3A). As the dose increased, the number of larval deaths increased, and $100 \%$ larval deaths were recorded at $10^{7} \mathrm{CFUs}$ (Figure 3A) and $10^{6} \mathrm{CFUs}$ (Figure 3B), at $24 \mathrm{hr}$ and $48 \mathrm{hr}$ respectively. The number of surviving larvae, injected with TTSS $^{-v e}$ strain, remained higher as compared to larvae injected with $\operatorname{TTSS}^{+\mathrm{ve}}$ strain, regardless of time and dose. (Figure 3). The comparative percentage of survival results indicates the G. mellonella is more susceptible to $\mathrm{TTSS}^{+\mathrm{ve}}$ non-O1/non-139 V. cholerae.

A

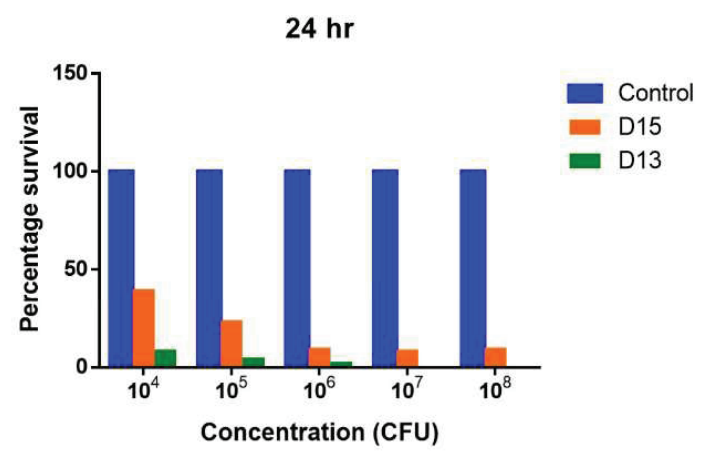

B

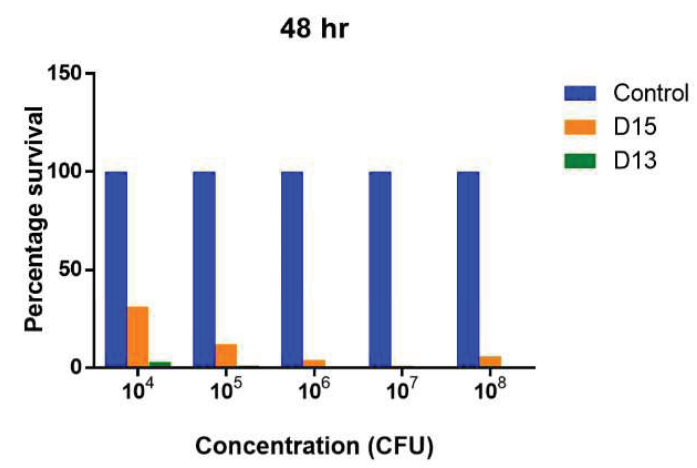

Figure 3: Comparison of killing potential of D-15 and D-13 against G. mellonella at different doses and time. CFUs, colony forming units.

\section{Discussion}

Vibrio cholerae associated with acute watery diarrhea has a substantial prevalence in Pakistan, since the disease is endemic in South Asia and some African and South American countries [4]. Cholera results in higher morbidity and mortality in children and the elderly population. Since $V$. cholerae is transmitted through fecal oral route so, increase in cases is 
observed during monsoon seasons often resulting in floods contaminating water reservoirs with cargo pathogens including $V$. cholerae. $V$. cholerae has a great variety of serogroups, among them only O1 and O139 cause epidemics because these strains encode the cholera toxin and other virulent arsenals which are responsible for characteristic cholera symptoms [24]. However, the non-O1/non-O139 strains also caused local outbreaks which are increasing day by day due to consumption of raw sea food or extra-intestinal infections from recreational waters [50]. Furthermore, these strains are acquiring more virulent characteristics which are helpful in evolving more pathogenic non-O1/non-O139 isolates posing higher levels of threats.

In this study, two non-O1/non-O139 $\mathrm{V}$. cholerae associated diarrheal cases with distinct clinical picture were analyzed to better understand the pathogenicity based on phenotypic and genomic characteristics. The study revealed that both non-O1/non-O139 isolates (D13 and D15) lacked the cholera toxin as well as toxin co-regulated pili which are the unique feature of toxigenic $V$. cholerae of $\mathrm{O} 1$ and $\mathrm{O} 139$ serogroups. However, D13 caused severe acute diarrheal disease whereas D15 caused mild diarrhea. Both the isolates possessed $r t x \mathrm{~A}$ and hemolysin $(h l y \mathrm{~A})$ as a majority of the non-agglutinating vibrios (nag) from other regions also shown to possess hemolysin $[38,39,41,42,51]$. Furthermore, both isolates possessed dth (gammathermostable hemolysisn), the product of which is similar to Tdh hemolysin of $V$. parahemolyticus, however $d t h$ role in disease has not been clearly elucidated among nonO1/non-O139 V. cholerae [52]. Moreover, key virulence players such as TcpA from vibrio pathogenicity island-1 (VPI-1) as well as other important members of VPI-1 such as toxT, toxR, acf, $\operatorname{tag} A$ and ald $A$ were not found. Partial or intact VPI-1 were found in some non-O1/non-O139 $V$. cholerae from some parts were isolated but this is usually not common. It has also been seen 
that presence of $t c p$ A or VPI- 1 in NAG vibrio may be associated with acquisition of $c t x$ phage in shaping up highly virulent non-O1/non-o139 in the environment [53].

VSP-1 and VSP-II found in the contemporary toxigenic $V$. cholerae O1 El Tor, usually absent in the six pandemic O1 $V$. cholerae and majority of the non-O1/non-o139 vibrio or environmental vibrios [41]. VSP-1 and VSP-2 are rarely present in non-O1/non-O139 isolates as majority of the studies did not find these islands in these vibrios or only encode some open reading frames in case of their existence $[41,43]$ and that was the case for D13 and D15 isolates as well for current study.

Both (D13 and D15) the strains showed a multiple antibiotic resistance phenotype with resistance to streptomycin, co-trimoxazole, nalidixic acid, ciprofloxacin, ceftaxime and ofloxacin compared to some recent studies where non-O1/non-O139 were susceptible to antibiotics [23, 54]. However, enhanced level of resistance to ampicillin, chloramphenicol, fluoroquinolones, cotrimoxazole have been reported by many studies [55-57] like the current study. However, the resistance pattern of these non-O1/non-O139 isolates was different from $\mathrm{O} 1$ isolates of clinical origin in Pakistan [58]. Genotypically, integrative and conjugative element (ICE-SXT) was absent in these NAG isolates which is now consistently encoded by $\mathrm{O} 1 \mathrm{El}$ tor. In addition, mobile genetic elements such as integrons encoding resistance to various antibiotics were nonexistent. However, for chloramphenicol resistance encoding florR was present similarly, sulfamethoxazole resistance encoding sul2 was also detected by PCR. The different drug profile from those of $\mathrm{O} 1 \mathrm{~V}$. cholerae suggested that empirical antibiotic cholera therapy is relevant for non-O1 infections and it must be revised. Moreover, there is also a threat of an increase in antibiotic resistance as, like other reservoirs, the marine and aquatic habitats are also under risk due to increased anthropogenic activities [59]. 
On the other hand, $c t x A$, ace, zot, $m s h A$, were absent in both isolates investigated during the current study. Previous studies also show that non-O1/non-O139 isolates usually do not produce cholera toxin unlike $\mathrm{O} 1$ and $\mathrm{O} 139$ [60]. However, these non-O1/non-O139 isolates may acquire cholera toxin carrying CTX phage and transfer of CTX phage from ctx positive to $c t x$ negative non-O1/non-O139 isolates [61].

The genome analysis in the TTSS gene cluster support the similarity of non-O1/nonO139 TTSS $V$. cholerae D13 with $V$. parahaemolyticus TTSS gene cluster [17]. The high similarity of TTSS gene cluster and organizations were found among $V$. cholerae D13 and $V$. parahaemolyticus in comparison with $V$. cholerae AM 19226. However, some genes are present only in $V$. parahaemolyticus while missing in $V$. cholerae D13, on the other hand, some genes are present only in $V$. cholerae D13 while missing in V. parahaemolyticus. Galleria melonella infection model analysis indicated that the presence of TTSS (D13) has contributed in enhancing its virulence compared to another non-O1 strain (D15) lacking TTSS and which somehow has been corroborated with the clinical picture of the patient as well.

At this time, we cannot say exactly what molecular mechanism of pathogenesis lead to frequent death of the G. mellonella larvae after infection with $\mathrm{TTSS}^{+\mathrm{ve}}$ nonO1/nonO139 but it may have contributed either directly or indirectly by allowing the production of other virulence factors or toxins such as RTX.

\section{Acknowledgements}

This work is partially supported by British Council through the Inspire Research support program for Universities (grant no. Sp 0019). 


\section{Appendix A}
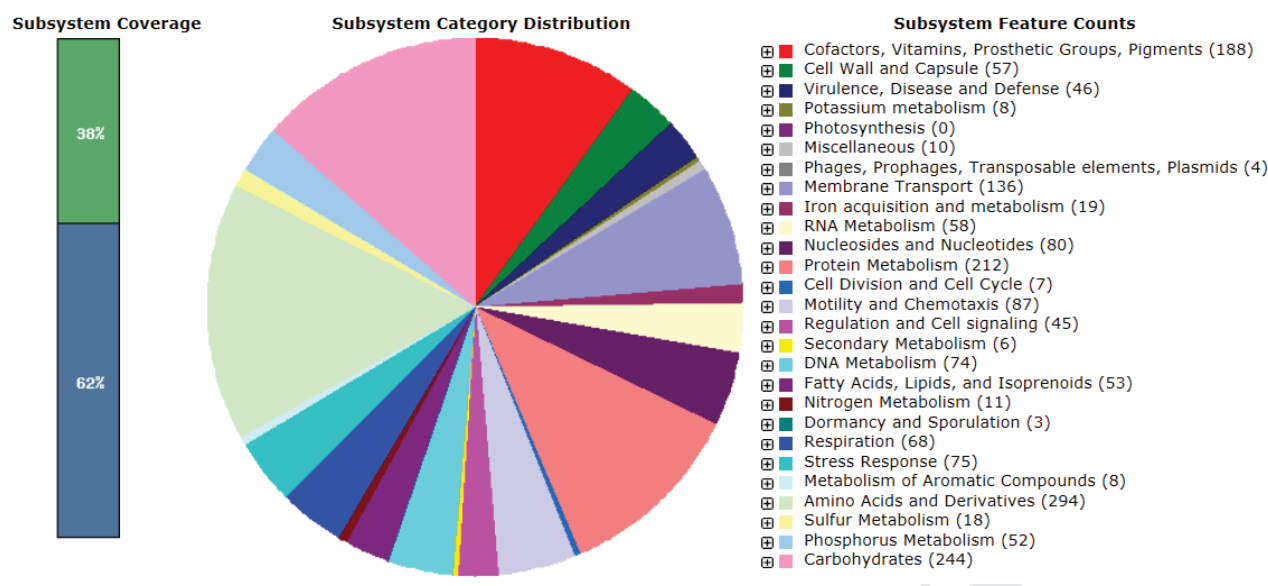

Figure A1: The Diagram of V. cholerae D13 based on Subsystem Category
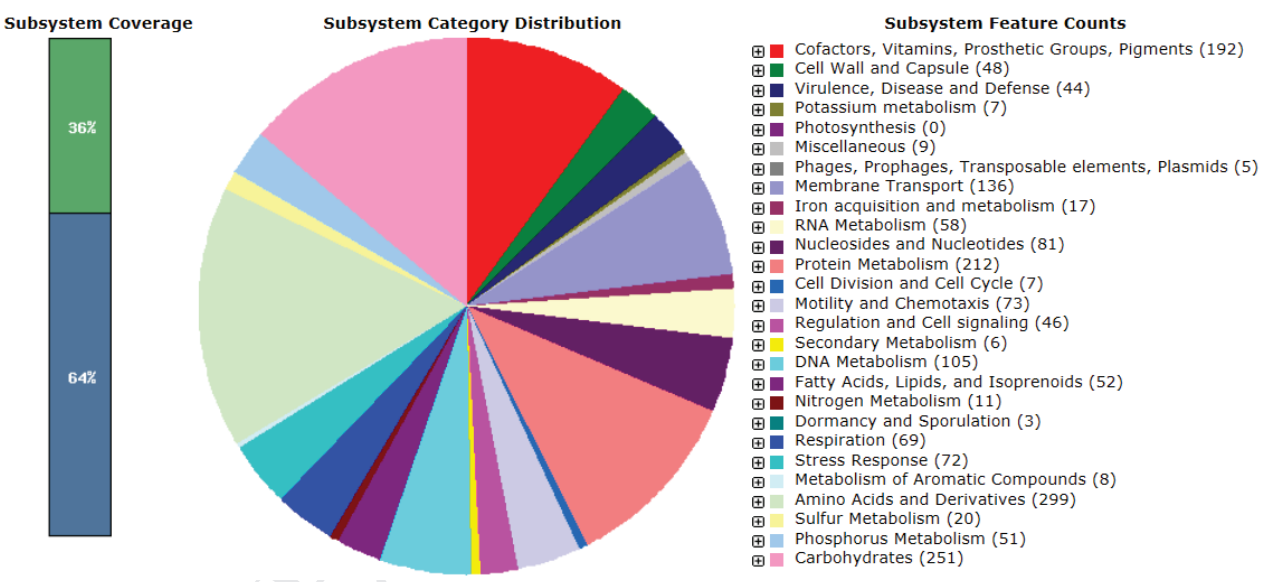

Figure A2: The Diagram of V. cholerae D15 based on Subsystem Category

\section{References:}

[1] Trucksis M, Michalski J, Deng YK, Kaper JB. The Vibrio cholerae genome contains two unique circular chromosomes. Proceedings of the National Academy of Sciences of the United States of America. (1998);95:14464-9.

[2] Wachsmuth IK, Blake PA, Olsvik Ø. Vibrio cholerae and cholera:molecular to global perspectives. Washington, D.C.: ASM Press; (1994). 
[3] Yamai S, Okitsu T, Shimada T, Katsube Y. Distribution of serogroups of Vibrio cholerae non-O1 non-O139 with specific reference to their ability to produce cholera toxin, and addition of novel serogroups. Kansenshogaku zasshi The Journal of the Japanese Association for Infectious Diseases. (1997);71:1037-45.

[4] Faruque SM, Chowdhury N, Kamruzzaman M, Dziejman M, Rahman MH, Sack DA, et al. Genetic diversity and virulence potential of environmental Vibrio cholerae population in a cholera-endemic area. Proc Natl Acad Sci U S A. (2004);101:2123-8.

[5] Trubiano JA, Lee JY, Valcanis M, Gregory J, Sutton BA, Holmes NE. Non-O1, non-O139 Vibrio cholerae bacteraemia in an Australian population. Intern Med J. (2014);44:508-11.

[6] Lu B, Zhou H, Li D, Li F, Zhu F, Cui Y, et al. The first case of bacteraemia due to nonO1/non-O139 Vibrio cholerae in a type 2 diabetes mellitus patient in mainland China. Int J Infect Dis. (2014);25:116-8.

[7] Waldor MK, Mekalanos JJ. Lysogenic conversion by a filamentous phage encoding cholera toxin. Science. (1996);272:1910-4.

[8] Karaolis DK, Johnson JA, Bailey CC, Boedeker EC, Kaper JB, Reeves PR. A Vibrio cholerae pathogenicity island associated with epidemic and pandemic strains. Proceedings of the National Academy of Sciences of the United States of America. (1998);95:3134-9.

[9] Bik EM, Bunschoten AE, Gouw RD, Mooi FR. Genesis of the novel epidemic Vibrio cholerae O139 strain: evidence for horizontal transfer of genes involved in polysaccharide synthesis. EMBO J. (1995);14:209-16.

[10] Hochhut B, Waldor MK. Site-specific integration of the conjugal Vibrio cholerae SXT element into prfC. Molecular microbiology. (1999);32:99-110.

[11] Haley BJ, Choi SY, Grim CJ, Onifade TJ, Cinar HN, Tall BD, et al. Genomic and phenotypic characterization of Vibrio cholerae non-O1 isolates from a US Gulf Coast cholera outbreak. PLoS One. (2014);9:e86264.

[12] Dalsgaard A, Serichantalergs O, Forslund A, Lin W, Mekalanos J, Mintz E, et al. Clinical and environmental isolates of Vibrio cholerae serogroup O141 carry the CTX phage and the genes encoding the toxin-coregulated pili. J Clin Microbiol. (2001);39:4086-92.

[13] Shin OS, Tam VC, Suzuki M, Ritchie JM, Bronson RT, Waldor MK, et al. Type III secretion is essential for the rapidly fatal diarrheal disease caused by non-O1, non-O139 Vibrio cholerae. MBio. (2011);2:e00106-11.

[14] Lin W, Fullner KJ, Clayton R, Sexton JA, Rogers MB, Calia KE, et al. Identification of a vibrio cholerae RTX toxin gene cluster that is tightly linked to the cholera toxin prophage. Proc Natl Acad Sci U S A. (1999);96:1071-6. 
[15] Cinar HN, Kothary M, Datta AR, Tall BD, Sprando R, Bilecen K, et al. Vibrio cholerae hemolysin is required for lethality, developmental delay, and intestinal vacuolation in Caenorhabditis elegans. PLoS One. (2010);5:e11558.

[16] Arita M, Takeda T, Honda T, Miwatani T. Purification and characterization of Vibrio cholerae non-O1 heat-stable enterotoxin. Infect Immun. (1986);52:45-9.

[17] Dziejman M, Serruto D, Tam VC, Sturtevant D, Diraphat P, Faruque SM, et al. Genomic characterization of non-O1, non-O139 Vibrio cholerae reveals genes for a type III secretion system. Proc Natl Acad Sci U S A. (2005);102:3465-70.

[18] Tam VC, Serruto D, Dziejman M, Brieher W, Mekalanos JJ. A type III secretion system in Vibrio cholerae translocates a formin/spire hybrid-like actin nucleator to promote intestinal colonization. Cell Host Microbe. (2007);1:95-107.

[19] Cornelis GR, Van Gijsegem F. Assembly and function of type III secretory systems. Annu Rev Microbiol. (2000);54:735-74.

[20] Mattoo S, Lee YM, Dixon JE. Interactions of bacterial effector proteins with host proteins. Curr Opin Immunol. (2007);19:392-401.

[21] Patel JC, Galan JE. Manipulation of the host actin cytoskeleton by Salmonella--all in the name of entry. Curr Opin Microbiol. (2005);8:10-5.

[22] Tam VC, Suzuki M, Coughlin M, Saslowsky D, Biswas K, Lencer WI, et al. Functional analysis of VopF activity required for colonization in Vibrio cholerae. MBio. (2010);1.

[23] Ceccarelli D, Chen A, Hasan NA, Rashed SM, Huq A, Colwell RR. Non-O1/non-O139 Vibrio cholerae carrying multiple virulence factors and V. cholerae O1 in the Chesapeake Bay, Maryland. Appl Environ Microbiol. (2015);81:1909-18.

[24] Dutta D, Chowdhury G, Pazhani GP, Guin S, Dutta S, Ghosh S, et al. Vibrio cholerae nonO1, non-O139 serogroups and cholera-like diarrhea, Kolkata, India. Emerg Infect Dis. (2013);19:464-7.

[25] Hoshino K, Yamasaki S, Mukhopadhyay AK, Chakraborty S, Basu A, Bhattacharya SK, et al. Development and evaluation of a multiplex PCR assay for rapid detection of toxigenic Vibrio cholerae O1 and O139. FEMS immunology and medical microbiology. (1998);20:201-7.

[26] Beaber JW, Hochhut B, Waldor MK. Genomic and functional analyses of SXT, an integrating antibiotic resistance gene transfer element derived from Vibrio cholerae. Journal of bacteriology. (2002);184:4259-69.

[27] Gubala AJ. Multiplex real-time PCR detection of Vibrio cholerae. Journal of microbiological methods. (2006);65:278-93. 
[28] Nandi B, Nandy RK, Mukhopadhyay S, Nair GB, Shimada T, Ghose AC. Rapid method for species-specific identification of Vibrio cholerae using primers targeted to the gene of outer membrane protein OmpW. Journal of clinical microbiology. (2000);38:4145-51.

[29] Ramachandran D, Bhanumathi R, Singh DV. Multiplex PCR for detection of antibiotic resistance genes and the SXT element: application in the characterization of Vibrio cholerae. Journal of medical microbiology. (2007);56:346-51.

[30] White PA, McIver CJ, Deng Y, Rawlinson WD. Characterisation of two new gene cassettes, aadA5 and dfrA17. FEMS microbiology letters. (2000);182:265-9.

[31] Kerrn MB, Klemmensen T, Frimodt-Moller N, Espersen F. Susceptibility of Danish Escherichia coli strains isolated from urinary tract infections and bacteraemia, and distribution of sul genes conferring sulphonamide resistance. The Journal of antimicrobial chemotherapy. (2002);50:513-6.

[32] Perreten V, Boerlin P. A new sulfonamide resistance gene (sul3) in Escherichia coli is widespread in the pig population of Switzerland. Antimicrobial agents and chemotherapy. (2003);47:1169-72.

[33] Cattoir V, Poirel L, Rotimi V, Soussy CJ, Nordmann P. Multiplex PCR for detection of plasmid-mediated quinolone resistance qnr genes in ESBL-producing enterobacterial isolates. The Journal of antimicrobial chemotherapy. (2007);60:394-7.

[34] Cattoir V, Weill FX, Poirel L, Fabre L, Soussy CJ, Nordmann P. Prevalence of qnr genes in Salmonella in France. The Journal of antimicrobial chemotherapy. (2007);59:751-4.

[35] Chuanchuen R, Pathanasophon P, Khemtong S, Wannaprasat W, Padungtod P. Susceptibilities to antimicrobials and disinfectants in Salmonella isolates obtained from poultry and swine in Thailand. The Journal of veterinary medical science / the Japanese Society of Veterinary Science. ((2008);70:595-601.

[36] Hochhut B, Lotfi Y, Mazel D, Faruque SM, Woodgate R, Waldor MK. Molecular analysis of antibiotic resistance gene clusters in vibrio cholerae O139 and O1 SXT constins. Antimicrobial agents and chemotherapy. (2001);45:2991-3000.

[37] Keasler SP, Hall RH. Detecting and biotyping Vibrio cholerae O1 with multiplex polymerase chain reaction. Lancet. (1993);341:1661.

[38] Rivera IN, Chun J, Huq A, Sack RB, Colwell RR. Genotypes associated with virulence in environmental isolates of Vibrio cholerae. Appl Environ Microbiol. (2001);67:2421-9.

[39] O'Shea YA, Finnan S, Reen FJ, Morrissey JP, O'Gara F, Boyd EF. The Vibrio seventh pandemic island-II is a $26.9 \mathrm{~kb}$ genomic island present in Vibrio cholerae El Tor and O139 serogroup isolates that shows homology to a $43.4 \mathrm{~kb}$ genomic island in $\mathrm{V}$. vulnificus. Microbiology. (2004);150:4053-63. 
[40] Schirmeister F, Dieckmann R, Bechlars S, Bier N, Faruque SM, Strauch E. Genetic and phenotypic analysis of Vibrio cholerae non-O1, non-O139 isolated from German and Austrian patients. Eur J Clin Microbiol Infect Dis. (2014);33:767-78.

[41] Rahman MH, Biswas K, Hossain MA, Sack RB, Mekalanos JJ, Faruque SM. Distribution of genes for virulence and ecological fitness among diverse Vibrio cholerae population in a cholera endemic area: tracking the evolution of pathogenic strains. DNA Cell Biol. (2008);27:347-55.

[42] Chatterjee S, Ghosh K, Raychoudhuri A, Chowdhury G, Bhattacharya MK, Mukhopadhyay AK, et al. Incidence, virulence factors, and clonality among clinical strains of non-O1, non-O139 Vibrio cholerae isolates from hospitalized diarrheal patients in Kolkata, India. Journal of clinical microbiology. 2009;47:1087-95.

[43] Purdy AE, Balch D, Lizarraga-Partida ML, Islam MS, Martinez-Urtaza J, Huq A, et al. Diversity and distribution of cholix toxin, a novel ADP-ribosylating factor from Vibrio cholerae. Environ Microbiol Rep. (2010);2:198-207.

[44] Zerbino DR, Birney E. Velvet: algorithms for de novo short read assembly using de Bruijn graphs. Genome research. (2008);18:821-9.

[45] Altschul SF, Gish W, Miller W, Myers EW, Lipman DJ. Basic local alignment search tool. Journal of molecular biology. (1990);215:403-10.

[46] Sullivan MJ, Petty NK, Beatson SA. Easyfig: a genome comparison visualizer. Bioinformatics. (2011);27:1009-10.

[47] Bokhari H, Ali A, Noreen Z, Thomson N, Wren BW. Galleria mellonella is low cost and suitable surrogate host for studying virulence of human pathogenic Vibrio cholerae. Gene. (2017);628:1-7.

[48] Champion OL, Wagley S, Titball RW. Galleria mellonella as a model host for microbiological and toxin research. Virulence. (2016);7:840-5.

[49] Winn WC, Allen S, Janda W, Koneman E, Procop G, Schreckenberger P, et al. Curved Gram-Negative Bacilli and Oxidase-Positive Fermenters: Campylobacteraceae and Vibrionaceae. In: EW K, editor. Koneman's Color Atlas and Textbook of Diagnostic Microbiology. 6th ed: Washington (USA): Lippincott Williams \& Wilkins; (2005). p. 408-28.

[50] Chen YT, Tang HJ, Chao CM, Lai CC. Clinical manifestations of non-O1 Vibrio cholerae infections. PLoS One. (2015);10:e116904.

[51] Singh DV, Matte MH, Matte GR, Jiang S, Sabeena F, Shukla BN, et al. Molecular analysis of Vibrio cholerae O1, O139, non-O1, and non-O139 strains: clonal relationships between clinical and environmental isolates. Appl Environ Microbiol. (2001);67:910-21. 
[52] Fallarino A, Attridge SR, Manning PA, Focareta T. Cloning and characterization of a novel haemolysin in Vibrio cholerae $\mathrm{O} 1$ that does not directly contribute to the virulence of the organism. Microbiology. (2002);148:2181-9.

[53] Klinzing DC, Choi SY, Hasan NA, et al. Hybrid Vibrio cholerae El Tor lacking SXT identified as the cause of a cholera outbreak in the Philippines. MBio. (2015);6(2):e00047-15.

[54] Chowdhury G, Joshi S, Bhattacharya S, Sekar U, Birajdar B, Bhattacharyya A, et al. Extraintestinal Infections Caused by Non-toxigenic Vibrio cholerae non-O1/non-O139. Front Microbiol. (2016);7:144.

[55] Bier N, Schwartz K, Guerra B, Strauch E. Survey on antimicrobial resistance patterns in Vibrio vulnificus and Vibrio cholerae non-O1/non-O139 in Germany reveals carbapenemaseproducing Vibrio cholerae in coastal waters. Front Microbiol. (2015);6:1179.

[56] Chandrasekhar MR, Krishna BV, Patil AB. Changing characteristics of Vibrio cholerae: emergence of multidrug resistance and non-O1, non-O139 serogroups. Southeast Asian J Trop Med Public Health. (2008);39:1092-7.

[57] Sellek RE, Niemcewicz M, Olsen JS, Bassy O, Lorenzo P, Marti L, et al. Phenotypic and genetic analyses of 111 clinical and environmental O1, O139, and non-O1/O139 Vibrio cholerae strains from different geographical areas. Epidemiol Infect. (2012);140:1389-99.

[58] Shah MA, Mutreja A, Thomson N, Baker S, Parkhill J, Dougan G, et al. Genomic Epidemiology of Vibrio cholerae O1 Associated with Floods, Pakistan, 2010. Emerg Infect Dis. (2014);20:13-20.

[59] Kumar PA, Patterson J, Karpagam P. Multiple antibiotic resistance profiles of Vibrio cholerae non-O1 and non-O139. Jpn J Infect Dis. (2009);62:230-2.

[60] Hasan NA, Ceccarelli D, Grim CJ, Taviani E, Choi J, Sadique A, et al. Distribution of virulence genes in clinical and environmental Vibrio cholerae strains in Bangladesh. Appl Environ Microbiol. (2013);79:5782-5.

[61] Haley BJ, Chen A, Grim CJ, Clark P, Diaz CM, Taviani E, et al. Vibrio cholerae in an Historically Cholera-Free Country. Environ Microbiol Rep. (2012);4:381-9. 


\section{Type III secretion system confers enhanced virulence in clinical non-O1/non- 0139 Vibrio cholerae}

\section{Highlights:}

- Type three secretion system (TTSS) is also considered one of the important virulent factors and during the current study we investigated the role of TTSS in association with non-O1/non-O139 clinical isolates.

- This article report that the presence of TTSS in non-O1/non-O139 V. cholerae clinical isolate (D13) from a child confers more virulence compared to the one lacking it (D15) in another clinical case during the small cholera epidemic.

- The antibiotic susceptibility profiles of D13 and D15 indicate that they are multiple drug resistance (MDR) isolates.

- The results revealed that that $\mathrm{TTSS}^{+\mathrm{ve}}$ isolate (D13) was more virulent compared to TTSS $^{-v e}$ isolate (D15).

- These finding was further validated by assessing virulence potential of both isolates using inexpensive G. mellonella infection model. 